\title{
Genetic Diversity of Fusarium proliferatum Populations from Maize, Onion, Rice and Sugarcane in Iran Based on Vegetative Compatibility Grouping
}

\author{
Alireza Alizadeh ${ }^{1}$, Mohammad Javan-Nikkhah ${ }^{1}$, Khalil-Berdi Fotouhifar ${ }^{1}$, Elahe Rabiee Motlagh ${ }^{2}$ and \\ Vahid Rahjoo ${ }^{3}$ \\ ${ }^{1}$ Department of Plant Pathology, University of Tehran, Karaj 31587-77871, Iran \\ ${ }^{2}$ Department of Plant Pathology, Faculty of Agriculture, Ferdowsi University of Mashhad, Mashhad-Iran \\ ${ }^{3}$ Department of Maize and Forage Crops Research, Seed and Plant Improvement Institute (SPII), P.O. Box 4119, Shahid \\ Fahmideh Bolv., 31585 Karaj, Iran
}

(Received on May 5, 2010; Accepted on May 26, 2010)

\begin{abstract}
Fusarium proliferatum is the causal agent of stalk and root rot disease of maize, foot rot disease of rice, basal and root rot disease of onion and knife cut disease of sugarcane in Iran. In recent years, incidence and severity of these diseases have been increased in Iran. Fifty seven $F$. proliferatum single-spore isolates collected from diseased maize, rice, onion and sugarcane plants at different areas were used to study genetic diversity by determination of vegetative compatibility groups (VCGs). Chlorate-resistant nitrate non-utilizing (nit) mutants were recovered from selected isolates of $\boldsymbol{F}$. proliferatum and used in complementation tests. All isolates in which both nit1 and NitM (or nit3) mutants were recovered, demonstrated self-compatibility. Vegetative compatibility tests by pairing nit mutants identified 30 VCGs among 57 isolates. Twenty-three isolates belonged to singlemember VCGs and the remaining 34 isolates, belonged to other seven multimember VCGs. Segregation of $F$. proliferatum isolates obtained from various area and host plants into different VCGs in Iran is reported for the first time. In this study, none of isolates obtained from rice complemented with any other isolates from onion and sugarcane and, non complementation occurred between onion and sugarcane isolates. Also, only one complementation occurred between one isolate of maize and one isolate of sugarcane and rice. Thus, a correlation between VCGs grouping and host preferences was founded. It is concluded that natural populations of $F$. proliferatum in Iran are probably genetically divergent and include isolates representing a potential risk for disease development.
\end{abstract}

Keywords : Fusarium proliferatum, genetic diversity, Nit mutant, vegetative compatibility grouping (VCG)

\footnotetext{
*Corresponding author.

Phone) $+0261-2818705, \quad$ FAX) $+98-261-2238529$

E-mail)jnikkhah@ut.ac.ir
}

Fusarium proliferatum (Matsushima) Nirenberg [Gibberella fujikuroi (Kuhlman) Samuels, Nirenberg \& Seifert, mating population $\mathrm{D}=$ Gibberella intermedia], is a prevalent pathogen of agriculturally important crops with wide host range. This pathogen can parasitize cultivated plants, and/or produce mycotoxins that pose serious hazards to human and animal health (Leslie \& Summerell, 2006).

F. proliferatum is the pathogen of some economically importance plants including rice, maize, citrus fruit, banana, orchids, sorghum, asparagus, pine, palm (Leslie \& Summerell, 2006), onion (Galvan et al., 2008) and sugarcane (Taherkhani, 1995). Particularly in Iran, it has been known as the causal agent of stalk and root rot of maize (Bujari et al., 1993, Ghiasian et al., 2004), foot rot disease of rice (Abbaszadeh et al., 2007), basal and root rot disease of onion (Rabiee motlagh, 2009) and knife cut disease of sugarcane (Taherkhani, 1995). More importantly, in recent years, the incidence and severity of these diseases have increased drastically in Iran. However, to the best of our knowledge, no comprehensive study on the Iranian $F$. proliferatum populations has been conducted so far.

Most recent assessments of fungal pathogens have used multilocus markers to detect populations (Milgroom, 1996). The ability of strains and/or species from geographically separated locations to recombine, poses the danger of introducing virulence and/ or toxigenic genes into local pathogen populations. One approach that has been used successfully in many fungi to examine population structure, clonality and the potential for gene flow is the study of vegetative incompatibility (Leslie, 1993). Fungal isolates that anastomose and form heterokaryons with each other are vegetatively compatible and are assigned to a single vegetative compatibility group (VCG) (Leslie, 1993). Members of the same VCG can form a stable heterokaryon, and share an identical set of alleles at about 10 vic loci (Leslie, 1993). Vegetative compatibility or anastomosis has been widely documented among several fungi and has proved to be a powerful tool in determining fungal genetic 
diversity (Correll et al., 1987, 1988; Nitzan et al., 2002; Puhalla, 1985).

F. proliferatum has not often been studied in depth from a population genetics perspective (Leslie \& Summerell 2006). Understanding the genetic structure of a population reflects its evolutionary history and its potential to evolve. However, in order to devise effective strategies to control pathogen growth, and to reduce the impact of disease, it is necessary to build knowledge on the genetic structure of pathogen population to be able to assess the level of pathogen variability and obtain additional insight into their population structure (McDonald, 2004; McDonald and McDermott, 1993). Therefore, knowledge of the genetic structure of the $F$. proliferatum populations might be useful in order to establish effective strategies for controlling the disease (McDonald, 2004). To date, most population genetics studies of Fusarium species have been conducted using the vegetative compatibility group as a marker for genotyping fungal isolates (Chulze et al., 2000; Danielsen et al., 1998).

In the previous study, presence of both mating type idiomorphs with favorable frequency and high effective population number among isolates recovered from different hosts of $F$. proliferatum, shows that there is potential for sexual reproduction within these populations and thus, an expansion of the genetic diversity by recombination (Alizadeh et al., 2009). Also, diversity for VCGs was very high in F. proliferatum isolates from rice recovered from different regions of Mazandaran province in Iran, where 26 different VCGs were found among 29 isolates (Alian et al., 2005).

The hypotheses of the current study were that there is VCG diversity within $F$. proliferatum populations and genetic exchange through sexual recombination frequently occurs in G. intermedia populations. However, weather different isolates of the fungus that infecting different hosts in same or different areas can exchange genetic materials through the anastomosis or they are different populations with distinct features that have own biological behavior. According to the results of the study we can clarily some aspects of their biology.

Our objective was to use VCGs identification to determine the genetic diversity among $F$. proliferatum isolates originating from different hosts in Iran.

\section{Materials and Methods}

Isolates. Isolates of Fusarium spp. were recovered from the stalks and seeds of 80 maize fields in 15 locations of 11 provinces of Iran (Ardabil, Azarbaijan, Fars, Gorgan, Hamedan, Isfahan, Kerman, Kermanshah, Mazandaran, Qazvin and Tehran) during the 2004-2005 growing seasons.
Other isolates include; 23 isolates from commercial rice field in Guilan and Mazandaran provinces at north of Iran (approximately more than $75 \%$ of rice growing areas are located in north of Iran), 20 isolates from onion in Khorasan province and 20 isolates from sugarcane from commercial sugarcane field in Khuzestan province. These isolates were identified using the morphological characteristics described by Leslie and Summerell (2006) and Nelson et al. (1983). PCR assay using species-specific primers confirmed morphological identification of $G$ intermedia isolates of maize and onion (Rahjoo et al., 2008; Rabiee motlagh, 2009). Isolates were obtained through the single spore subcultures and then were stored in filter paper at $-20^{\circ} \mathrm{C}$. These pure cultures were used as the straining inoculums for all subsequent tests. Fifty-seven isolates including; 20 isolates from maize, 20 isolates from rice, 12 isolates from sugarcane and five isolates from onion were selected for VCG determination, which was based on complementation reaction between NitM (or nit3) and nit1 mutants on minimal medium.

Media. In an initial test, chlorate-resistant mutants were generated on minimal agar medium with chlorate (MMC), Potato dexterose agar medium with chlorate (PDC), Czapek-Dox agar medium with chlorate and water agar medium amended by chlorate (WAC). Minimal medium (MM) was prepared by adding $2 \mathrm{~g}$ of $\mathrm{NaNO}_{3}$ to $1 \mathrm{~L}$ basal medium (Correll et al., 1987). MMC was prepared by adding 1-6 g L-asparagine, $2 \mathrm{~g} \mathrm{NaNO}_{3}$, and $30 \mathrm{~g} \mathrm{KClO}_{3}$ to $1 \mathrm{~L}$ of basal medium. PDC was prepared by adding $39 \mathrm{~g}$ $\mathrm{PDA}$, and $15 \mathrm{~g} \mathrm{KCIO}_{3}$ to $1 \mathrm{~L}$ distilled water. $\mathrm{CZC}$ was prepared by adding $39 \mathrm{~g}$ Czapek-Dox and $30 \mathrm{~g} \mathrm{KClO}_{3}$ to $1 \mathrm{~L}$ distilled water and WAC was prepared by adding $20 \mathrm{~g}$ agar and $30 \mathrm{~g} \mathrm{KClO}_{3}$ to $1 \mathrm{~L}$ distilled water. Subsequently chlorate-resistant mutants were generated on MMC containing $30 \mathrm{~g} \mathrm{KClO}_{3}$.

Recovery of nit mutants. Recovery of nit mutants was done according to the methods described by Klittich and Leslie (1988a). Mycelial discs (1-2 $\left.\mathrm{mm}^{3}\right)$ from each isolate were placed on minimal medium plus $3 \% \mathrm{KClO}_{3}$ for mutant selection. Plates were incubated at $24-25^{\circ} \mathrm{C}$ in dark and were examined weekly for the appearance of fast growing sectors. The sectors grow at a visibly faster rate than do the parental culture. This difference may result since wild-type growth may be limited due to the reduction of chlorate to toxic chlorite by nitrate reductase (Solomonson \& Vennesland, 1972). Approximately 20-40 chlorate-resistant sectors from each fungal isolates were selected on MMC and transferred to MM. Transfers that grew as expansive but thin mycelial colonies were considered to be nit mutants. 
Nit mutant phenotypes. The physiological phenotypes of nit mutants were investigated by growing them on media containing one of five different nitrogen sources, following the Correll et al. (1987) method.

Complementation test. VCG grouping was based on complementation reaction between NitM (or nit3) and nit1 mutants on minimal medium. Pairings to test vegetative compatibility were made in plates (10 $\mathrm{cm}$ in diameter) as previously described by Klittich and Leslie (1988b). Isolates were paired in all possible pairwise combinations and with a positive control. Pairs of isolates that exhibited vigorous aerial growth at the contact zone of the two nit mutant, indicating the formation of a heterokaryon, were determined as vegetative compatible and were assigned to the same VCG. Otherwise, isolates were defined as belonging to different VCGs.

\section{Results}

Nit mutants isolation. All 57 isolates of F. proliferatum spontaneously produced chlorate resistant sectors on MMC. All of them grew on MM as thin mycelium with no aerial mycelia (nit mutants) because of their inability to utilize nitrate as a sole nitrogen source. The majority of recovered chlorate resistant sectors were unable to utilize nitrate as a sole nitrogen source and consequently grew as thin expansive colonies with no aerial mycelia in MM. There was a marked difference in the rate at which sectoring occurred and in the recovery of nit mutants on MMC. A few sectors that were recovered from each strain were chlorate resistant, but able to utilize nitrate.

Nit mutant phenotype identification. Nit mutant phenotype was identified after growth of isolates on media that contained one of five different nitrogen sources. The nit mutants could be divided into three phenotypic classes. The nit mutants with wild-type growth on both nitrite and hypoxanthine-containing media were classified as nitl; those with wild-type growth on medium with hypoxanthine or nitrite as the sole $\mathrm{N}$ source were identified as nit 3 and NitM, respectively. The majority of nit mutants recovered on all medium were nit 1 mutants. (Table 1). However, the frequency of recovered nitl mutants was higher on PDC than on other mediums. Consequently, the relative frequency of nitM mutants recovered was considerably higher on MMC than on other media. Also, the highest relative frequency of nit 3 was observed on MMC. Among the nit mutants, the nit 1 class composed $79 \%$ of the total number of mutants, while nitM and nit 3 mutants composed $8 \%$ and $13 \%$ of the total number of mutants, respectively (Table 1).
Table 1. Frequency and phenotype of ninrate nonutilizing (nit) mutants recovered from four media

\begin{tabular}{|c|c|c|c|c|}
\hline \multirow{2}{*}{ Medium $^{\mathrm{a}}$} & \multirow{2}{*}{$n i t^{\mathrm{b}}(\%)$} & \multicolumn{3}{|c|}{ nit mutants classes ${ }^{c}$} \\
\hline & & nit1 (\%) & $\operatorname{nitM}(\%)$ & nit3 $(\%)$ \\
\hline PDC & 92 & 90 & 5 & 5 \\
\hline MMC & 89 & 64 & 15 & 21 \\
\hline $\mathrm{CZC}$ & 93 & 78 & 8 & 14 \\
\hline WAC & 78 & 86 & 2 & 12 \\
\hline Total & 88 & 79 & 8 & 13 \\
\hline
\end{tabular}

${ }^{\mathrm{a}} \mathrm{PDC}=$ potato-dexterose agar medium plus chlorate, $\mathrm{MMC}=$ minimal agar medium plus chlorate, $\mathrm{CZC}=$ Czapek-Dox agar chlorate plus chlorate, $\mathrm{WAC}=$ water agar medium plus chlorate.

${ }^{\mathrm{b}}$ Percent of chlorate-resistant sectors that grew as thin expansive colonies on minimal medium.

${ }^{c}$ Nit mutant phenotypes determined according to growth on basal medium amended with different nitrogen sources.

Complementation test. All isolates in which were recovered both nitl and NitM (or nit3) mutants demonstrated self-compatibility. Based on their positive complementation reactions, thirty VCGs were identified among 57 isolates, of these 23 groups had only one member, and the remaining 34 isolates belonged to seven multimember VCGs (Table 2 ), accounting for a genetic diversity (number of VCGs/ number of isolates) of 0.54 . VCG1 was the largest and the most frequent group in Iran and consisted of 20 isolates, VCG2 included four isolates whereas the other five multimember VCGs each had two members.

\section{Discussion}

Genetic characterization of prevailing F. proliferatum populations is important for effective management of the given diseases. Examination of selected isolates from Iran for vegetative compatibility and sexual fertility (Alizadeh et al., 2009) further confirm the status of the F. proliferatum isolates that originating from various hosts in Iran. According to the previous study, based on morphological characteristics and sexual fertility crosses, the 142 isolates were assigned to "D" mating population of G. fujikuroi. Furthermore, morphological identification of these isolates as $G$. intermedia was confirmed by sexual crosses. The usability of the diagnostic PCR method for mating type identification was tested on $142 \mathrm{~F}$. proliferatum isolates obtained from maize, rice, onion and sugarcane plants from various areas of Iran. Both MAT-1 and MAT-2 individuals were identified among these all isolates (Alizadeh et al., 2009). These results showed that there is potential for sexual reproduction within these populations and thus, an expansion of the genetic diversity by recombination.

Distribution of $F$. proliferatum isolates obtained from various area and host plants into different VCGs in Iran is 
Table 2. Characterization of nit mutants from F. proliferatum isolated from different host plants in different locations of Iran.

\begin{tabular}{|c|c|c|c|c|c|c|c|c|}
\hline \multirow{2}{*}{$\begin{array}{l}\text { Isolate } \\
\text { (no. })^{\mathrm{a}}\end{array}$} & \multirow{2}{*}{ Location $^{\mathrm{b}}$} & \multirow{2}{*}{$\begin{array}{l}\text { Host/Infected } \\
\text { tissue }\end{array}$} & \multirow{2}{*}{ Year } & \multirow{2}{*}{$\begin{array}{c}\text { Mating } \\
\text { type }^{\mathrm{C}}\end{array}$} & \multicolumn{3}{|c|}{ Phenotype of nit mutants } & \multirow{2}{*}{$\begin{array}{l}\text { VCG } \\
\text { Group }^{d}\end{array}$} \\
\hline & & & & & $\mathrm{A}$ & B & $\mathrm{C}$ & \\
\hline 1 & Ardabil & Maize- ear & 2005 & MAT-2 & nit1 & nit3 & NitM & 1 \\
\hline 2 & Ardabil & Maize- ear & 2005 & MAT-1 & nit1 & - & NitM & 3 \\
\hline 3 & Ardabil & Maize- ear & 2005 & MAT-1 & nit1 & - & - & 8 \\
\hline 4 & Ardabil & Maize-ear & 2005 & MAT-2 & nit1 & nit3 & NitM & 1 \\
\hline 5 & Ardabil & Maize-ear & 2005 & MAT-2 & nit1 & nit3 & - & 1 \\
\hline 6 & East-Azerbaijan & Maize-stalk & 2005 & MAT-1 & nit1 & nit3 & - & 9 \\
\hline 7 & Fars & Maize-ear & 2005 & MAT-1 & nit1 & nit3 & - & 10 \\
\hline 8 & Fars & Maize-ear & 2005 & MAT-1 & nit1 & - & NitM & 3 \\
\hline 9 & Fars & Maize-ear & 2005 & MAT-1 & nit1 & - & NitM & 1 \\
\hline 10 & Gorgan & Maize-ear & 2005 & MAT-1 & nit1 & nit3 & NitM & 1 \\
\hline 11 & Hamedan & Maize-ear & 2005 & MAT-1 & nit1 & nit3 & NitM & 1 \\
\hline 12 & Hamedan & Maize- ear & 2005 & MAT-2 & nit1 & - & NitM & 1 \\
\hline 13 & Isfahan & Maize- ear & 2005 & MAT-2 & nit1 & nit3 & - & 2 \\
\hline 14 & Isfahan & Maize- ear & 2005 & MAT-2 & nit1 & nit3 & - & 4 \\
\hline 15 & Kerman & Maize-ear & 2005 & MAT-1 & nit1 & nit3 & NitM & 1 \\
\hline 16 & Kermanshah & Maize- ear & 2005 & MAT-1 & nit1 & nit3 & - & 4 \\
\hline 17 & Mzandaran & Maize-ear & 2005 & MAT-2 & nit1 & - & - & 11 \\
\hline 18 & Mzandaran & Maize-ear & 2005 & MAT-1 & nit1 & nit3 & - & 12 \\
\hline 19 & Qazvin & Maize-ear & 2005 & MAT-1 & nit1 & - & NitM & 1 \\
\hline 20 & Tehran & Maize-ear & 2005 & MAT-1 & nit1 & nit3 & - & 13 \\
\hline 21 & Guilan & Rice-foot & 2004 & MAT-1 & nit1 & nit3 & NitM & 2 \\
\hline 22 & Guilan & Rice-foot & 2004 & MAT-1 & nit1 & - & NitM & 2 \\
\hline 23 & Guilan & Rice-foot & 2004 & MAT-1 & nit1 & - & NitM & 14 \\
\hline 24 & Guilan & Rice-foot & 2004 & MAT-1 & nit1 & nit3 & - & 15 \\
\hline 25 & Guilan & Rice-foot & 2004 & MAT-2 & nit1 & nit3 & - & 16 \\
\hline 26 & Guilan & Rice-foot & 2004 & MAT-2 & nit1 & nit3 & - & 17 \\
\hline 27 & Mazandaran & Rice-foot & 2004 & MAT-1 & nit1 & nit3 & NitM & 2 \\
\hline 28 & Mazandaran & Rice-foot & 2004 & MAT-2 & nit1 & nit3 & - & 5 \\
\hline 29 & Mazandaran & Rice-foot & 2004 & MAT-2 & nit1 & nit3 & NitM & 5 \\
\hline 30 & Mazandaran & Rice-foot & 2004 & MAT-1 & nit1 & - & NitM & 6 \\
\hline 31 & Mazandaran & Rice-foot & 2004 & MAT-2 & nit1 & nit3 & NitM & 6 \\
\hline 32 & Mazandaran & Rice-foot & 2004 & MAT-2 & nit1 & nit3 & - & 7 \\
\hline 33 & Mazandaran & Rice-foot & 2004 & MAT-2 & nit1 & - & NitM & 7 \\
\hline 34 & Mazandaran & Rice-foot & 2004 & MAT-1 & nit1 & nit3 & NitM & 18 \\
\hline 35 & Mazandaran & Rice-foot & 2004 & MAT-2 & nit1 & nit3 & - & 19 \\
\hline 36 & Mazandaran & Rice-foot & 2004 & MAT-2 & nit1 & - & NitM & 20 \\
\hline 37 & Mazandaran & Rice-foot & 2004 & MAT-1 & nit1 & nit3 & - & 21 \\
\hline 38 & Mazandaran & Rice-foot & 2004 & MAT-2 & nit1 & nit3 & NitM & 22 \\
\hline 39 & Mazandaran & Rice-foot & 2004 & MAT-2 & nit1 & nit3 & - & 23 \\
\hline 40 & Mazandaran & Rice-foot & 2004 & MAT-2 & nit1 & nit3 & - & 24 \\
\hline 41 & Khuzestan & Sugarcane & 2003 & MAT-1 & nit1 & nit3 & - & 25 \\
\hline 42 & Khuzestan & Sugarcane-stem & 2003 & MAT-2 & nit1 & - & NitM & 1 \\
\hline 43 & Khuzestan & Sugarcane-stem & 2003 & MAT-1 & nit1 & - & NitM & 1 \\
\hline 44 & Khuzestan & Sugarcane-stem & 2003 & MAT-1 & nit1 & nit3 & - & 26 \\
\hline 45 & Khuzestan & Sugarcane-stem & 2003 & MAT-1 & nit1 & - & NitM & 1 \\
\hline 46 & Khuzestan & Sugarcane-stem & 2003 & MAT-2 & nit1 & nit3 & - & 1 \\
\hline 47 & Khuzestan & Sugarcane-stem & 2003 & MAT-1 & nit1 & - & NitM & 1 \\
\hline 48 & Khuzestan & Sugarcane-stem & 2003 & MAT-2 & nit1 & nit3 & NitM & 1 \\
\hline
\end{tabular}


Table 2. Characterization of nit mutants from F. proliferatum isolated from different host plants in different locations of Iran.

\begin{tabular}{|c|c|c|c|c|c|c|c|c|}
\hline \multirow{2}{*}{$\begin{array}{l}\text { Isolate } \\
\text { (no.) })^{\mathrm{a}}\end{array}$} & \multirow{2}{*}{ Location $^{\mathrm{b}}$} & \multirow{2}{*}{$\begin{array}{c}\text { Host/Infected } \\
\text { tissue }\end{array}$} & \multirow{2}{*}{ Year } & \multirow{2}{*}{$\begin{array}{l}\text { Mating } \\
\text { type }^{\mathrm{C}}\end{array}$} & \multicolumn{3}{|c|}{ Phenotype of nit mutants } & \multirow{2}{*}{ VCG } \\
\hline & & & & & A & B & $\mathrm{C}$ & \\
\hline 49 & Khuzestan & Sugarcane-stem & 2003 & MAT-2 & nit1 & nit3 & - & 27 \\
\hline 50 & Khuzestan & Sugarcane-stem & 2003 & MAT-1 & nit1 & - & NitM & 28 \\
\hline 51 & Khuzestan & Sugarcane-stem & 2003 & MAT-2 & nit1 & nit3 & - & 29 \\
\hline 52 & Khuzestan & Sugarcane-stem & 2003 & MAT-1 & nit1 & - & - & 30 \\
\hline 53 & North Khorasan & Onion-root & 2007 & MAT-1 & nit1 & - & NitM & 1 \\
\hline 54 & North Khorasan & Onion-scale & 2007 & MAT-1 & nit1 & nit3 & NitM & 1 \\
\hline 55 & North Khorasan & Onion-root & 2007 & MAT-1 & nit1 & - & NitM & 1 \\
\hline 56 & North Khorasan & Onion-root & 2007 & MAT-1 & nit1 & - & NitM & 1 \\
\hline 57 & Rzavi Khorasan & Onion-basal & 2007 & MAT-1 & nit1 & nit3 & NitM & 1 \\
\hline
\end{tabular}

${ }^{\mathrm{a}}$ Isolate number.

${ }^{\mathrm{b}}$ Locations are different provinces of Iran.

${ }^{\mathrm{c}}$ Mating type data for examined isolates obtained from the study conducted by Alizadeh et al. (2009).

${ }^{\mathrm{d}} \mathrm{VCG}=$ vegetative compatibility groups (Total 57 isolates grouped in 30 VCGs based on vegetative compatibility tests by pairing nit mutants).

reported for the first time. We identified 30 VCGs among 57 tested isolates. Isolates belonging to VCG1 were collected from eight provinces, indicating that genetic variation across geographic locations occurs in Iran, confirming results obtained in previous studies (Desjardins, 2003; Bargen et al., 2009). VCG1 included isolates from maize, onion and sugarcane host plants and VCG2 included isolates from maize and rice. Each of VCG 3, VCG 4 and VCG 5 included two isolates from maize and each of VCG 6 and VCG 7 included two isolates from rice. Beside those, 23 isolates grouped in 23 VCGs that each of them had only one member. This result was similar to those of Alian et al. (2005) that 26 VCG groups identified among 29 isolates. Of those, 23 VCGs were represented each by a single isolates and 6 isolates belonged to three multimember groups, each containing two isolates. In this study, none of isolates from rice complemented with any other isolates from onion and sugarcane. Also, non complementation occurred between onion and sugarcane isolates. In VCG1 only one complementation occurred between one isolate from maize and sugarcane isolates. Also, in VCG2 only one isolate from maize complemented with rice isolates. Therefore except some cases, host preferences observed in complementation tests and assignment of isolates to VCG groups. Missing relationships between DNA fingerprint groups and origin of isolates or other characteristic features are often described for different Fusarium species, which causes various plant diseases. For instance, Abd-Elsalam et al. (2003) were able to correlate diverse fingerprint patterns of $F$. semitectum isolates with morphological characteristics of fungal cultures, but found no correlation with host genotype or geographic origin. Patino et al. (2001) investigated thirty-three isolates of $F$. verticillioides isolated from diverse origins and hosts. Analysis of the IGS region by PCR-RFLP discriminated two groups of isolates based on fumonisin production and host preferences. Moretti et al., (2004) reported that, isolates of $F$. verticillioides from banana showed different traits such as pathogenecity, toxin profile, fertility and AFLP fingerprint than members of the same isolates from maize. In the present study, a correlation between VCGs grouping and host preferences was founded. Also, individuals with both mating types, MAT-1 and $M A T-2$, were found in the multimember VCGs groups indicating that those isolates in same VCGs would not be clones of one another but different individuals.

Isolates from maize belonging to VCG 1 were collected from eight provinces, also indicating that genetic variation across geographic locations occurs in Iran. Since clonal spread by rain splash or wind over the large distances is unlikely, the same VCG genotype was probably introduced into different sites as seed-borne inoculums. Infected maize seed must be a significant vehicle for the dissemination of pathogen genotypes throughout Iran. In addition, we think that fungal populations including VCG1 may be adapted to a maize genotype which was commonly cultivated in 2004 -2005 .

Molecular phylogenetic analyses of strains from diverse hosts and geographic areas initiated by Desjardins (2003) exhibited high diversity within $F$. proliferatum ( $G$. intermedia) isolates, which were recovered from asparagus spears from different locations in Austria and Germany, confirmed considerable genetic variability (Bargen et al., 2009). Alian et al. (2005) investigated twenty-nine isolates of $F$. proliferatum recovered from rice from Mazandaran province in Iran and 26 VCG groups identified among 29 isolates. Mohammadian (2008) reported high genetic diver- 
sity of $F$. proliferatum isolates obtained from maize and rice in Iran using RAPD molecular marker. In addition, AFLP analysis of the $F$. proliferatum populations obtained from various hosts and geographical areas showed that natural populations of $F$. proliferatum in Iran are genetically divergent and include isolates representing a potential risk for disease development (Alizadeh, 2010).

Present study showed the high genetic diversity within Iranian populations of $F$. proliferatum from different plant hosts in Iran. These results are in agreement with results from previous studies on $F$. proliferatum, demonstrating that the populations of this fungus are genotypically highly diverse in Iran (Alian et al., 2005; Mohammadian, 2008).

Preliminary results on sexual fertility revealed that occurrence of sexual recombination and genetic variations are possible in $G$. intermedia populations in Iran (Alizadeh et al., 2009). Abbaszadeh et al. (2007) recorded that isolates of $F$. proliferatum have the ability to reproduce both sexually and asexually in Iran. Extensive sexual recombination should, however, increase the level of diversity in the $G$. intermedia populations by recombining the alleles present into new combination. Meiotic recombination can generate and maintain genetic variation and results in the reassortment of genes that govern traits such as virulence or toxin production (Cumagun et al., 2002).

Results obtained from other studies (Mohammadian, 2008; Alian et al., 2005; Abbaszadeh et al., 2007; Alizadeh et al., 2009; Alizadeh, 2010) and high degree of VCGs polymorphism in this study supports the assumption that the genotypic diversity of $F$. proliferatum is primarily caused by recombination during the sexual reproduction. And also, the worldwide distribution of $F$. proliferatum and the wide range of hosts from which it has been isolated can support the existence of variability within this species.

In order to improve understanding of the structure of populations, additional isolates from all hosts growing regions should be tested and neutral DNA markers such as AFLP would generate further information on the genetic structure of $F$. proliferatum populations in Iran.

\section{Acknowledgments}

This study was financially supported by grant of the Iranian High Council for Research. We gratefully acknowledge the University of Tehran for providing materials.

\section{References}

Abbaszadeh, M., Nikkhah, M. J., Padasht Dehkayi, F., Mousanejad, S. 2007. Sexual fertility and mating types of Gibberella fujikuroi species complex, the cause of Bakanae disease and foot rot in Guilan province, Iran. Iran. J. Agri. Sci. 38:685-
692.

Abd-Elsalam, K. A., Schnieder, F., Asran-Amal, A., Khalil, M. S. and Vereet, J. A. 2003. Intra-species genomic groups in Fusarium semitectum and their correlation with origin and cultural characteristics. Z Pflanzenkr Pflanzenschutz 110:409-418.

Alian, A., Javan-Nikkhah, M., Aminian, H. and Khosravi, V. 2005. Study on Genetic Diversity of Gibberella intermedia, The Common Causal Agent of Rice Bakanae Disease in Mazandaran Province, based on Vegetative Compatibility Groups. Iran. J. Agri. Sci. 38:685-692.

Alizadeh, A. 2010. Study on genetic diversity of Gibberella intermedia isolates recovered from maize, rice and sugarcane using AFLPs molecular marker and vegetative compatibility groups (VCGs). M.Sc. thesis, University of Tehran, Iran.

Alizadeh, A., Javan-Nikkhah, M., Fotouhifar, Kh., Mohammadian, E., Rabiee Motlagh, E. and Ghazanfari, K. 2009. Fertility status and PCR based identification of mating type alleles in Gibberella intermedia populations from maize, rice, onion and sugarcane in Iran. Asian Mycological Congress (AMC 2009), Taiwan.

Bargen, V. S., Martinez, O., Schadock, I., Eisold, A. M., Gossmann, M. and Buttner, C. 2009. Genetic Variability of Phytopathogenic Fusarium proliferatum Associated with Crown Rot in Asparagus officinalis. J. Phytopathol. 157: 446-456.

Bujari, J., Ershad, D., 1993. An investigation on corn seed mycoflora. Iran. J. Plant Pathol. 29:13-17.

Campbell, C. L., Leslie, J. F. and Farrokhi-Nejad, R. 1992. Genetic diversity of Fusarium moniliforme in seed from two maize cultivars. Phytopathology 82:1082.

Correll, J. C., Klittich, C. J. R. and Leslie, J. F. 1987. Nitrate nonutilizing mutants of Fusarium oxysporum and their use in vegetative compatibility test. Phytopathology 77:1640-1646.

Chulze, S. N., Ramirez, M. L., Torres, A. and Leslie, J. F. 2000. Genetic variation in Fusarium section Liseola from no-till maize in Argentina. Appl. Environ. Microbiol. 66:5312-5315.

Cumagun, C. J. R., Bowden, R. L. and Miedaner, T. 2002. Segregation of aggressiveness in a crossing population of Fusarium graminearum. J. Appl. Genet. 43:39-44.

Danielsen, S., Meyer, U. M. and Funck Jensen, D. 1998. Genetic characteristics of Fusarium verticillioides isolates from maize in Costa Rica. Plant Pathol. 44:615-622.

Desjardins, A. E. 2003. Giberella from A(venaceae to Z(eae). Anmu. Rev. Phytopathol. 41:177-198.

Farrokhi-Nejad, R. and Leslie, J. F. 1990. Vegetative compatibility group diversity with populations of Fusarium moniliforme isolates from corn seed. Phytopathology 80:1043.

Galván, G A., Koning-Boucoiran, C. F. S., Koopman, W. J. M., Burger-Meijer, K., González, P. H., Waalwijk, C., Kik, C. and Scholten, O. E. 2008. Genetic variation among Fusarium isolates from onion and resistance to Fusarium basal rot in related Allium species. Eur. J. Plant Pathol. 121:499-512.

Ghiasian, S. A., Kord-Bacheh, P., Rezayat, S. M., Maghsood, A. H. and Taherkhani, H. 2004. Mycoflora of Iranian maize harvested in the main production areas in 2000. Mycopathologia 158:113-121.

Klittich, C. J. R. and Leslie, J. F. 1988a. Nitrate reduction mutants 
of Fusarium moniliforme (Gibberella fujikuroi). Genetics 118: 417-423.

Klittich, C. J. R. and Leslie, J. F. 1988b. Multiwell plates for complementation tests of Fusarium. Fungal Gen. Newsletter 35:21-22.

Leslie, J. F. 1991. Mating populations in Gibberella fujikuroi (Fusarium section Liseola). Phytopathology 81:1058-1060.

Leslie, J. F. 1993. Fungal vegetative compatibility. Annu. Rev. Phytopathol. 31:127-151.

Leslie, J. F. 1995. Gibberella fujikuroi: available populations and variable traits. Can. J. Bot. 73:282-291.

Leslie, J. F. and Klein, K. K. 1996. Female fertility and matingtype effects on effective population size in filamentous fungi. Genetics 144:557-576.

Leslie, J. F. and Summerell, B. A. 2006. The Fusarium Laboratory Manual. Blackwell Publishing Professional, Ames, USA. $388 \mathrm{pp}$.

McDonald, B. A. 2004. Population genetics of plant pathogens. The Plant Health Instructor DOI, 10.1094/PHI-A-20040524-01.

McDonald, B. A. and McDermott, J. M. 1993. Population genetics of plant pathogenic fungi. Bioscience 43:311-319.

Milgroom, M. G 1996. Recombination and the multilocus structure of fungal populations. Annu. Rev. Phytopathol. 34:457477.

Mohammadian, E. 2008. Study on genetic diversity of Gibberella moniliformis and G. intermedia from corn and rice and identi- fication of frequency of MAT locus alleles based on PCR. M.Sc. thesis, University of Tehran, Karaj, Iran.

Nelson, P. E., Toussoun, T. A. and Marasas, W. F. O. 1983. Fusarium species: an illustrated manual for identification. The Pennsylvania State University Press, University Park, USA. 203 pp.

Nitzan, N., Hazanovsky, M., Tal, M. and Tsror, L. 2002. Vegetative Compatibility Groups in Colletotrichum coccodes, the Causal Agent of Black Dot on Potato. Phytopathology 92: 827-832

Puhalla, J. E. 1985. Classification of strains of Fusarium oxysporum on the basis of vegetative compatibility. Can. J. Bot. 63:179183

Rabiee motlagh, E. 2009. Study on phytopatpgenic fungi of root and bulb of onion in Razavi and north Khorasan provinces. M.Sc. thesis, ferdowsi University of Mashhad, Mashhad, Iran.

Rahjoo, V., Zad, J., Javan-Nikkhah, M., Mirzadi Gohari, A., Okhovvat, S. M., Bihamta, M. R., Razzaghian, J. and Klemsdal, S. S. 2008. Molecular and morphological identification of fusarium isolated from maize ears in Iran. J. Plant Pathol. 90:463-468.

Solomonson, L. P. and Vennesland, B. 1972. Nitrate reductase and chlorate toxicity in Chlorella vulgaris Beijerinck. Plant physiol. 50:421-424.

Taherkhani, K. 1995. Study of sugarcane fusariosis diseases in khouzestan province. M.Sc. thesis, University of Tarbiat Modarres, Tehran, Iran. 